\title{
Chaos in Accretion Disk Dynamos?
}

\author{
ULF TORKELSSON \\ Lund Observatory, Box 43, S-22100 Lund, Sweden \\ and
}

AXEL BRANDENBURG

HAO/NCAR, P.O. Box 3000, Boulder, CO 80307, USA

\begin{abstract}
Accretion disks appear to be favourable places for dynamo action, because of their strong differential rotation. A simple estimate of the strength of an accretion disk dynamo indicates that it will be highly nonlinear. In spite of this, most studies hitherto have assumed a linear model for the dynamo. Here we investigate nonlinear, axisymmetric mean-field dynamos in accretion disks in order to study the route to chaotic solutions in certain parameter regimes. We find a sequence of bifurcations that lead eventually to chaos. Finally, the physical significance of these results is discussed.
\end{abstract}

\section{INTRODUCTION}

Accretion, i.e. the inflow of matter onto a massive object, is a common phenomenon in astrophysics. There are two limiting cases of accretion, spherical and disk accretion. Accretion disks are important for the understanding of several types of objects, e.g. cataclysmic variables and X-ray binaries. The infalling matter forms a disk, because it possesses too much angular momentum to be able to settle directly onto the central object. Thus the main function of the disk is to allow the accreting mass to dissipate its angular momentum. The major problem in accretion disk theory is to understand this transport of angular momentum. It is usually assumed that some type of turbulent viscosity is responsible. However, if magnetic fields are present (e.g. due to a dynamo effect), the magnetic torque can achieve the transport of angular momentum.

Because the rotation of the disk is close to Keplerian, the disk will rapidly wind up a frozen-in magnetic field, thus requiring only a small amount of turbulence to drive an $\alpha \Omega$-dynamo. By simple order-of-magnitude estimates it can be demonstrated that the dynamo numbers for accretion disks are much larger than required to excite the dynamo [1]. It is therefore of interest to study the characteristics of nonlinear dynamos in accretion disks, which is the aim of this paper. In Section 2 we present our model and computational method, and then in Section 3 we demonstrate how the dynamo depends on the dynamo number, i.e. the strength of the turbulence in the accretion disk. Section 4 contains a discussion of our results. 


\section{DISK MODEL AND COMPUTATIONAL METHOD}

We assume that the evolution of a mean magnetic field $\mathbf{B}$ can be described by the $\alpha \Omega$-dynamo equations

$$
\frac{\partial \mathbf{B}}{\partial t}=\nabla \times\left(\mathbf{u} \times \mathbf{B}+\alpha \mathbf{B}-\eta_{\mathrm{t}} \mu_{0} \mathbf{J}\right), \quad \nabla \cdot \mathbf{B}=0,
$$

where $\mathbf{u}$ is the mean velocity, $\alpha$ describes the generation of the mean magnetic field through helical (cyclonic) turbulence, $\eta_{\mathrm{t}}$ is the turbulent magnetic diffusivity, $\mu_{0}$ the magnetic permeability of free space, and $\mathbf{J}=\nabla \times \mathbf{B} / \mu_{0}$ the mean electric current. Throughout this paper we assume Keplerian rotation in the main part of the disk, and set $\mathbf{u}=0$ outside the disk.

Equation (1) is solved numerically in spherical polar coordinates $(r, \theta, \phi)$, using the same method as in [1]. The outer radius of the computational grid, $R$, is the unit of length, and the inner radius is at $r_{0}=0.05$, where a perfect conductor is assumed. On $r=R$ the solution is matched to a potential field outside. Assuming axisymmetry, $\partial / \partial \phi=0$, we split equation (1) into two equations for the $B_{\phi}$ and $A_{\phi}$.fields, where $A_{\phi}$ is the $\phi$-component of the vector potential.

The geometry of the disk is specified in cylindrical polar coordinates $(\omega, \phi, z)$. The half-thickness of the disk is $z_{0}=0.2$, the inner edge at $\varpi_{0}=0.15$, and the disk extends to $r=R$. Outside the disk, but still inside the computational sphere, we assume an enhanced magnetic diffusivity $\eta_{\text {out }}$, relative to the value $\eta_{\text {disk }}$ inside the disk in order to simulate a surrounding vacuum. We assume that $\eta_{\text {disk }}$ is equal to the kinematic viscosity, for which Shakura and Sunyaev [2] proposed the simple prescription $v_{\text {disk }}=\alpha_{\mathrm{ss}} c_{\mathrm{s}} z_{0}$, where $c_{\mathrm{s}}$ is the speed of sound, and $\alpha_{\mathrm{SS}}$ a dimensionless parameter less than unity. Assuming hydrostatic equilibrium in the vertical direction, we have $c_{\mathrm{s}}^{2} \approx\left(G M / \omega^{2}\right)\left(z_{0} / \sigma\right) z_{0}=\Omega_{\mathrm{Kepl}}^{2} z_{0}^{2}$ for a thin disk, where $\Omega_{\text {Kepl }}$ is the Keplerian angular velocity around an object of mass $M$. For most of a thin accretion disk we can make the approximation $z_{0} \propto \varpi$ [2] giving $\eta_{\text {disk }}=v_{\text {disk }} \propto \varpi^{1 / 2}$. Nondimensional units are defined by putting $\eta_{\text {out }}=1$ and $\eta_{\text {disk }}(1)=0.1$.

The $\alpha$-effect describes the effect of cyclonic motion on the magnetic field, and it exists in the presence of both rotation and stratification. Since the Coriolis force changes sign at the disk plane, the simplest estimate, is $\alpha \approx \Omega z$ (see [3] for an explicit derivation). Furthermore, there is a feedback from the Lorentz force that quenches $\alpha$ with increasing $B$. Thus a simple form for the $\alpha$-effect is

$$
\alpha=\alpha_{0} \frac{z}{R} \frac{\Omega}{\Omega\left(\omega_{0}\right) 1+\alpha_{B} B^{2}},
$$

where $\alpha_{0}$ is a characteristic value of $\alpha, \alpha_{B}$ is the inverse equipartition field energy density, and $\Omega\left(\varpi_{0}\right)$ is the Keplerian angular velocity at the inner edge of the disk. The sign of $\alpha_{0}$ is defined by the relative importance of the stratification of density and turbulent velocity. If there is only stratification in density, rising elements expand and get distorted by the Coriolis force in such a way that $\alpha_{0}>0$ (see Fig 9.1 in [4]). However, if for some reason the gradient in the turbulent intensity is sufficiently strong, the sign of $\alpha_{0}$ can be reversed. Thus it is necessary to investigate both positive and negative values of $\alpha_{0}$.

For simplicity we set $\alpha_{B}=$ const. $=1$, but see [1] for results with nonuniform $\alpha_{B}$. The dynamo is described by two dimensionless numbers, $C_{\Omega}=\Omega\left(\varpi_{0}\right) R^{2} / \eta_{\text {out }}$, and $C_{\alpha}=$ $\alpha_{0} R / \eta_{\text {out }}$, and in the limit $C_{\Omega} \gg C_{\alpha}$ solely by the product $C_{\Omega} C_{\alpha}$. Note that we define the dynamo numbers and the unit of time, $[t]=R^{2} / \eta_{\text {out }}$, in terms of the magnetic diffusivity outside the disk, which is different from [1]. 


\section{PROPERTIES OF NONLINEAR SOLUTIONS}

In order to investigate the properties of the magnetic field, we solve equation (1) for $C_{\Omega}=25,000$ and different values of $C_{\alpha}$. In Figs 1 and 2 we plot the energies of saturated solutions and the frequencies of the oscillatory solutions as a function of $C_{\alpha}$. We denote the solutions of even (odd) parity as S0 (A0), where ' 0 ' stands for axisymmetry. The calculations have been carried out either in one quadrant of the meridional plane, imposing the parity on the solution, or in two quadrants and not prescribing a priori the parity of the magnetic field. The size of the grid is $41 \times 41$ for one quadrant and $41 \times 81$ for two. For solutions of mixed parity we use the parameter

$$
P=\frac{E^{(\mathrm{S})}-E^{(\mathrm{A})}}{E^{(\mathrm{S})}+E^{(\mathrm{A})}},
$$

where $E^{(\mathrm{S})}$ and $E^{(\mathrm{A})}$ are the energies of the symmetric and antisymmetric parts of the magnetic field, respectively, to characterize the solutions.

\subsection{Positive $C_{\alpha}$}

As expected from linear calculations, a steady S0-solution appears for $C_{\alpha}>0.0013$, but more surprisingly it dies away again before an oscillatory A0-solution emerges (Fig. 1). For $0.0063<C_{\alpha}<0.015$ only the $\mathbf{B}=0$ solution is stable. This feature is, however, sensitive to the type of nonlinearity adopted. If the field is limited by the effect of magnetic buoyancy [1], the field does not vanish in the gap. Another example is the somewhat unphysical
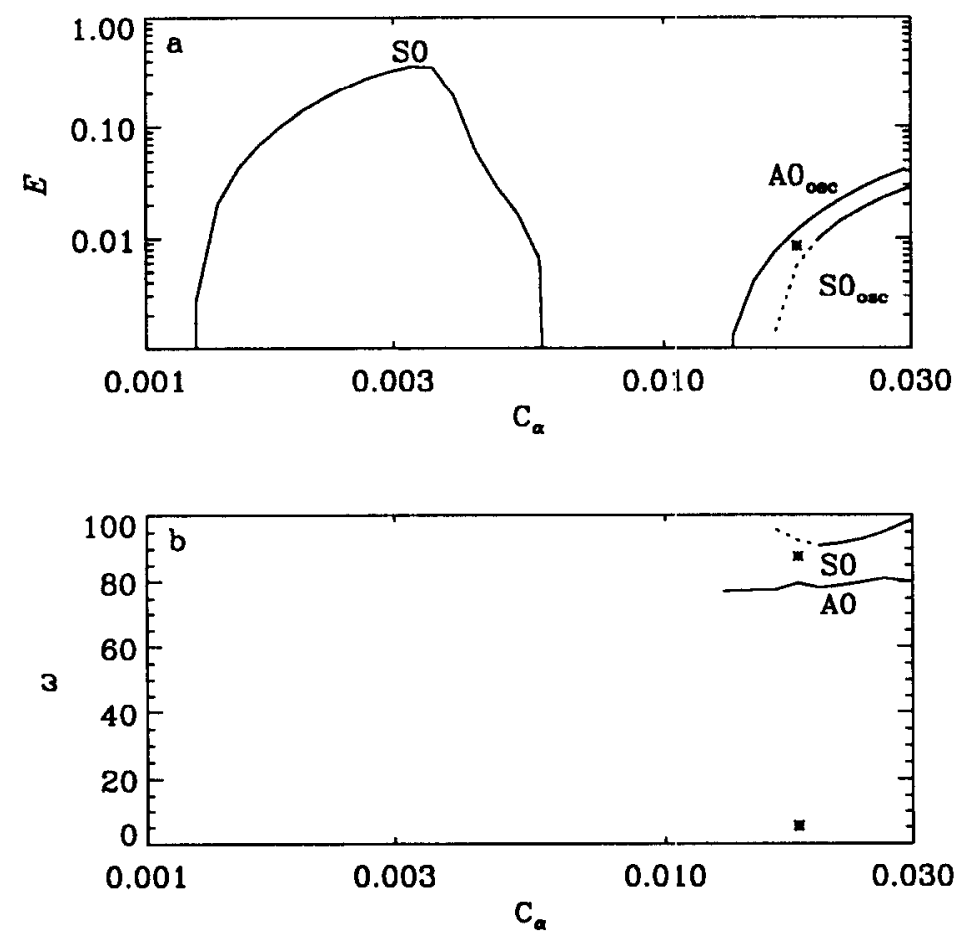

Fig. 1. (a) Energy and (b) angular frequency as a function of $C_{\alpha}>0$. A.0 and S0 stand for axisymmetric solutions of negative and positive parity, respectively. The subscript 'osc' in (a) denotes oscillatory solutions. Dashed lines mark unstable solutions. The stars represent the values for a doubly periodic solution at $C_{\alpha}=0.018$ oscillating in both $E$ and $P$. 

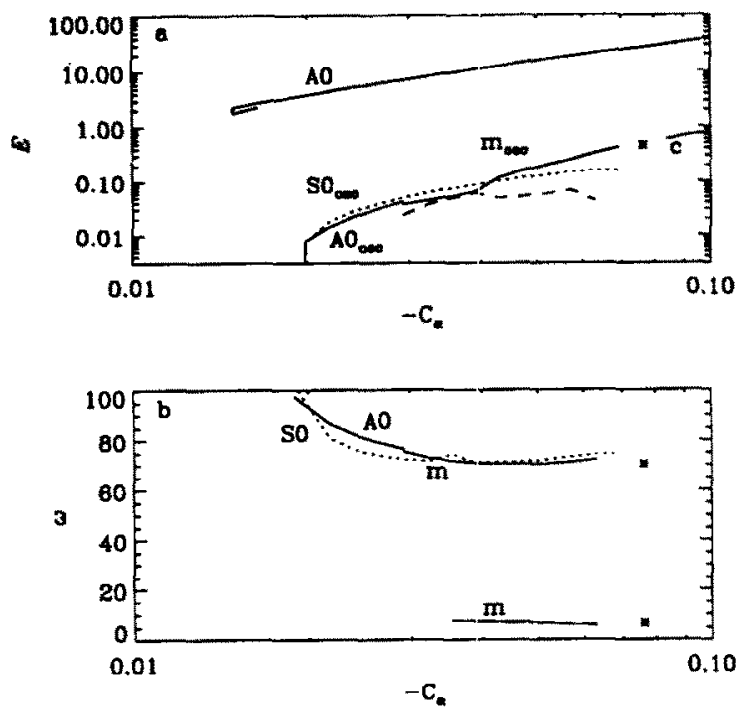

Fig. 2. (a) Energy and (b) angular frequency as a function of $C_{\alpha}<0 . \mathrm{m}$ denotes a solution of mixed parity oscillating on one or several frequencies in both $E$ and $P$, and $c$ denotes a solution varying chaotically in both $E$ and $P$, however also the solution at the right end of the m-branch is chaotic. The dashed line shows the minimum energy for the mixed-mode solutions. The stars stand for a doubly periodic solution of even parity at $C_{\alpha}=-0.076$. All other symbols have the same meaning as in Fig. 1 . The mixed parity solution at $C_{\alpha}=-0.063$ is triple-periodic, but the third frequency $\omega=1.5$ has not been included in the plot.

quenching via the total energy, i.e. $\alpha \propto 1 /(1+E)[5,6]$, in which case the energy always increases linearly with $C_{\alpha}$, and thus no gap is possible.

For larger $C_{\alpha}$ there appears firstly an oscillatory $A 0$-solution $\left(C_{\alpha}>0.013\right)$, and then also an oscillatory $\mathrm{S} 0\left(C_{\alpha}>0.016\right)$ which, however, is initially unstable to perturbations of opposite parity. (We are able to calculate the unstable solution of pure parity by constraining the calculations to one quadrant and imposing the parity on the numerical solution.) This S0-solution becomes stable shortly after the appearance of a doubly periodic solution oscillating in both $E$ and $P$ with the angular frequencies $\omega=87.6$ and 5.4. At this point $\left(C_{\alpha}=0.018\right)$ the frequency difference of these two solutions is minimal. Beyond this point both the $\mathrm{S} 0$ - and $\mathrm{A} 0$-solutions are stable, and initial seed fields of mixed parity will evolve to either one of the two solutions. There is a watershed between two possibilities: at $C_{\alpha}=0.02$, for example, solutions with $P>-0.118$ evolve to $\mathrm{S} 0$, and those with $P<-0.118$ to A0.

\subsection{Negative $C_{\alpha}$}

The situation is more complicated for $C_{\alpha}<0$ (Fig. 2). The first nontrivial solution is steady and has $P=-1\left(\left|C_{\alpha}\right|>0.015\right)$. This solution was not found in the corresponding linear calculations [1]. When we decreased $\left|C_{\alpha}\right|$ to 0.014 , the magnetic field decayed towards zero, but if $\left|C_{\alpha}\right|$ was put equal to 0.015 before the magnetic field strength had gone down by too much, we found a new steady solution at a smaller magnetic energy, which is the lower branch of the curve. However, for $\left|C_{\alpha}\right|>0.016$ this solution becomes unstable, and the magnetic field evolves to the state corresponding to the upper branch of the curve.

Unfortunately, it is impossible to trace unstable solutions of the same parity using a time-stepping method, but we expect that this solution originates from a subcritical 
bifurcation of the trivial solution. It is interesting to note that for disks with slightly different geometrical properties (smaller $z_{0}$ or larger $\varpi_{0}$ ), this solution emerges via an ordinary supercritical bifurcation [1].

At $C_{\alpha} \approx-0.020$ there are supercritical bifurcations to oscillatory S0- and $\mathrm{A} 0$-solutions. The two solutions have similar energies and frequencies, but the S0-solution is unstable. Later the A0-solution becomes oscillatory in both $E$ and $P$, and after some time also doubly periodic (Figs 3, 4). The phase portrait and Poincaré map (Fig. 4) for $C_{\alpha}=-0.039$ indicate that the trajectory of this solution lies on a $\mathrm{T}^{2}$ torus. From the power spectrum it is also evident that there are two frequencies with most of the power being at the higher frequency. The amplitude of the oscillations in $E$ reaches a minimum close to $C_{\alpha}=$ -0.036 , whereas the amplitude of $P$ continuously increases. At $C_{\alpha}=-0.063$ a third (smaller) frequency, appears at $\omega=1.5$ (Fig. 5), suggesting that the solution evolves on a $\mathrm{T}^{3}$ torus. Now the strongest power is at the intermediate frequency. The phase portrait and the Poincaré map of the solution shows rather regulat behaviour, suggesting that the solution is perhaps not a $\mathrm{T}^{3}$ torus, but a more complicated $\mathrm{T}^{2}$ torus (Fig. 6).

Later there first appears a chaotic solution for a narrow interval around -0.069 , and then at $C_{\alpha}=-0.076$ the solution is again doubly periodic with the angular frequencies $\omega=70.4$ and 6.6 , but it has changed character to be of pure quadrupole parity and is only oscillating in the magnetic energy. Increasing the dynamo number to $C_{\alpha}=-0.084$ leads finally to a chaotic solution, with variations both in $E$ and $P$ (Fig. 7). The phase portrait and the Poincare map of this solution is plotted in Fig. 3 . Note that the previously sharp peaks in the power spectrum have broadened to two less well-defined humps.
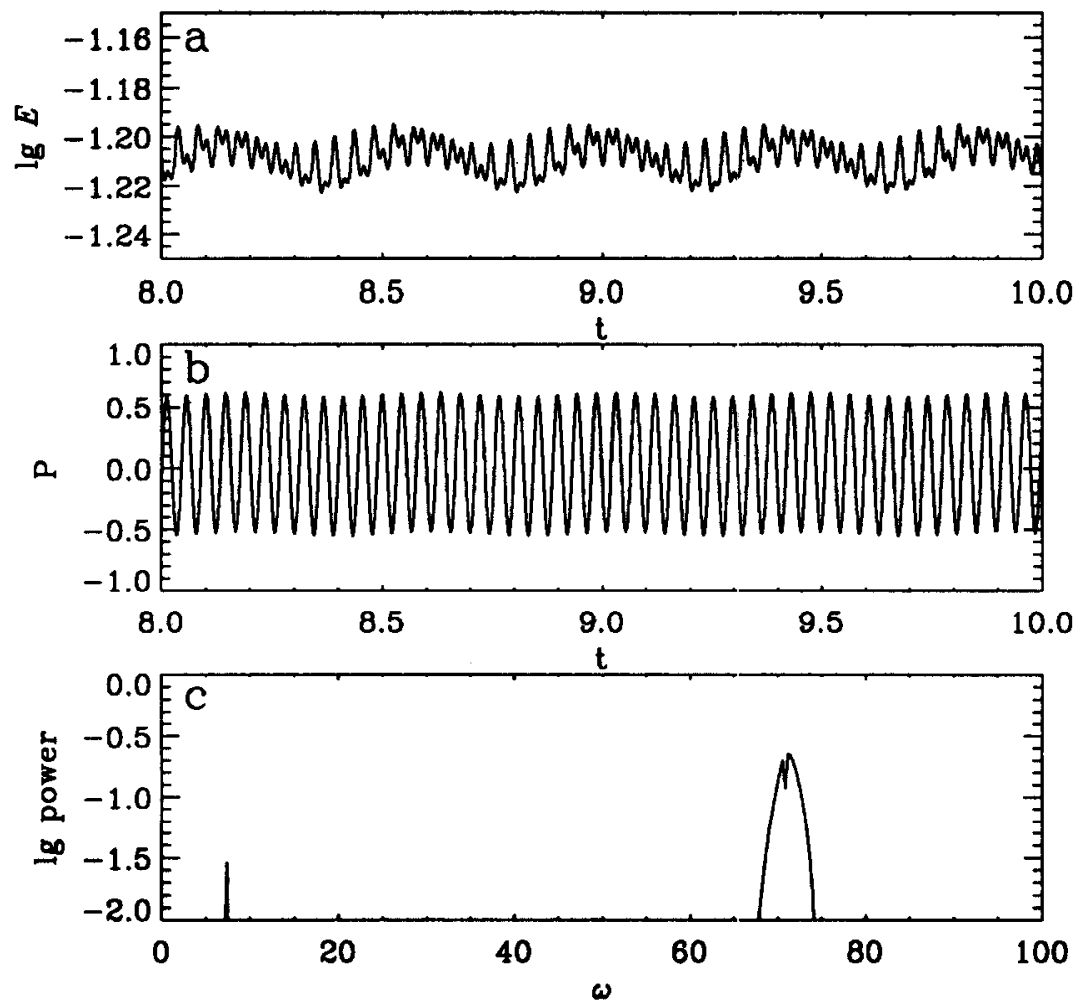

Fig. 3. The evolutions of $E$ (a) and $P$ (b) for a doubly periodic solution with $C_{\alpha}=-0.039$. The power spectrum of $E$ is displayed in (c). Note the two peaks at $\omega=7$ and $\omega=71$. 

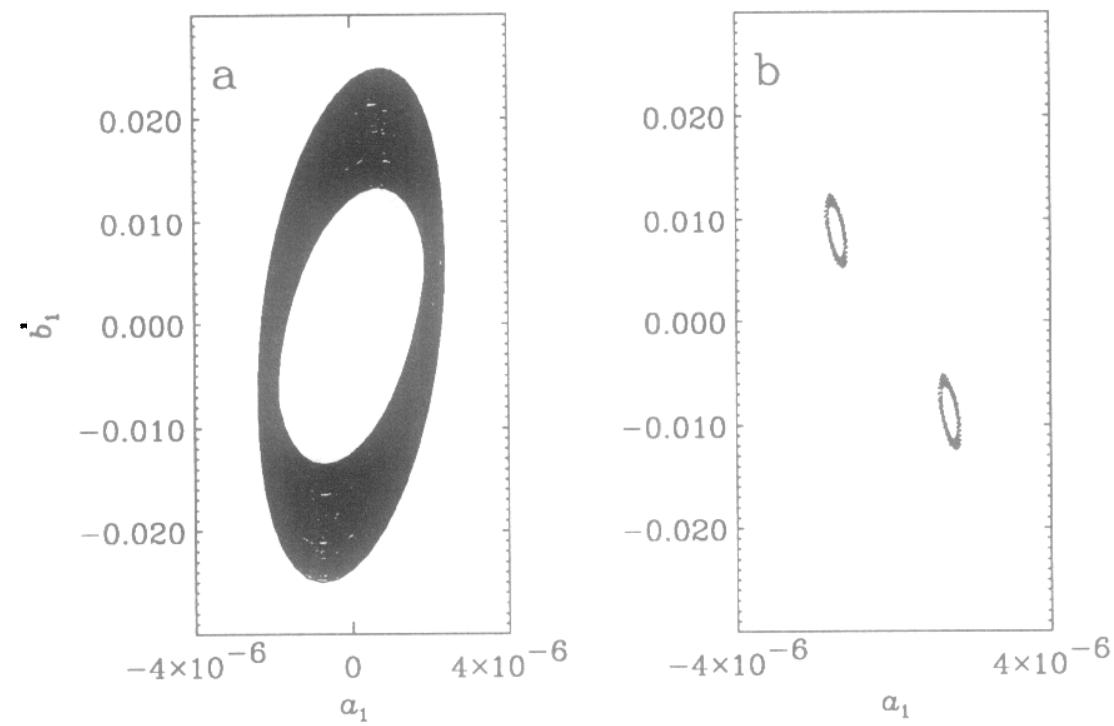

Fig. 4. (a) The phase portrait of the solution for $C_{\alpha}=-0.039$ (see Fig. 3) projected onto the $A_{\phi}-B_{\phi}$-planc of a point at $(\sigma, z)=(0.52,-0.04)$ in the grid. (b) The Poincaré map of this solution. We plot the points where the $B_{\phi^{-f i e l d}}$ at $(0.52,0.04)$ goes through zero.
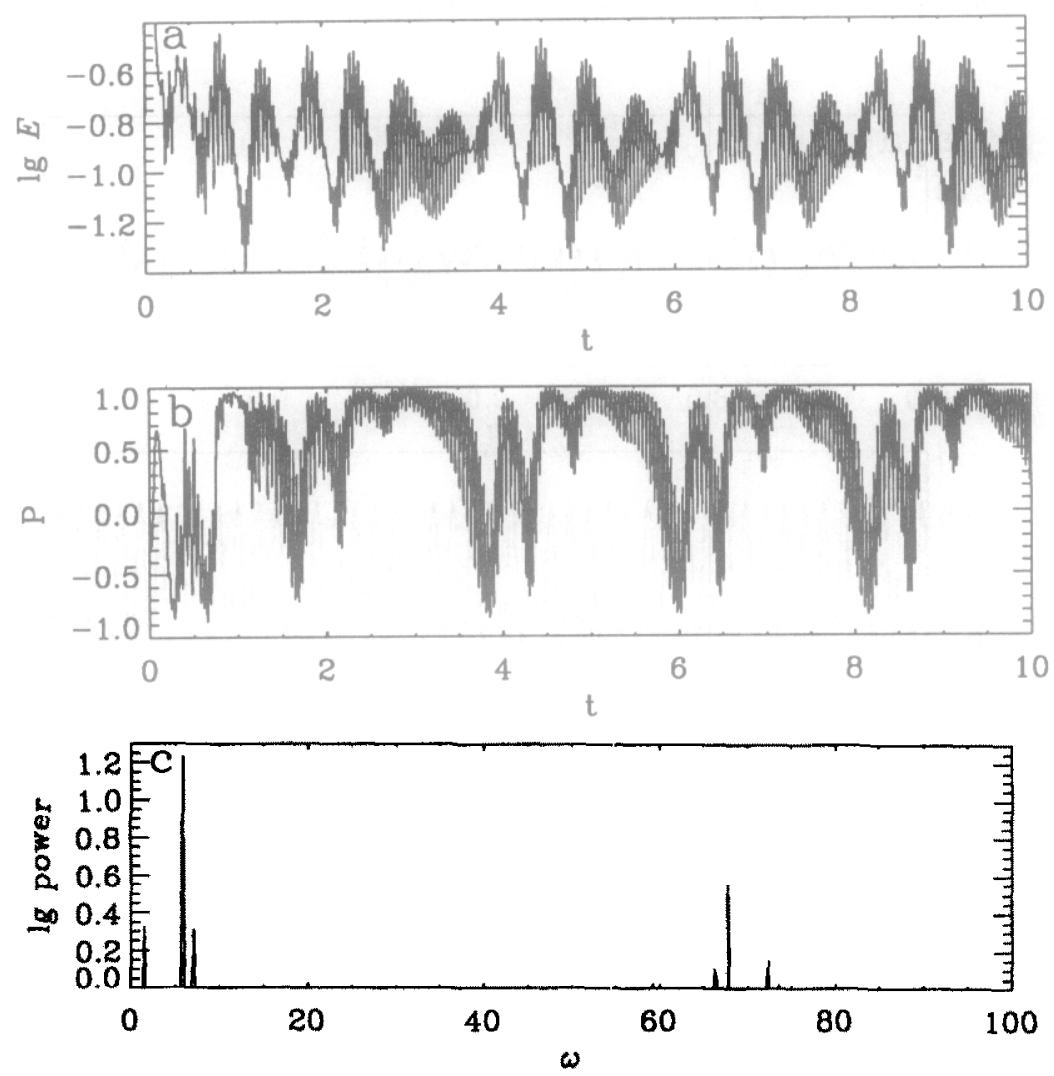

Fig. 5. The variations in $E$ (a) and $P$ (b) for a triply-periodic dynamo solution with $C_{\alpha}=-0.063$. The power spectrum of the oscillations in $E$ are displayed in (c). Note the occurrence of three distinct frequencies at $\omega_{1}=1.5, \omega_{2}=6$, and $\omega_{3}=68$. (The peak at about 7.5 results from the superpositition of $\omega_{1}$ and $\omega_{2}$.) 

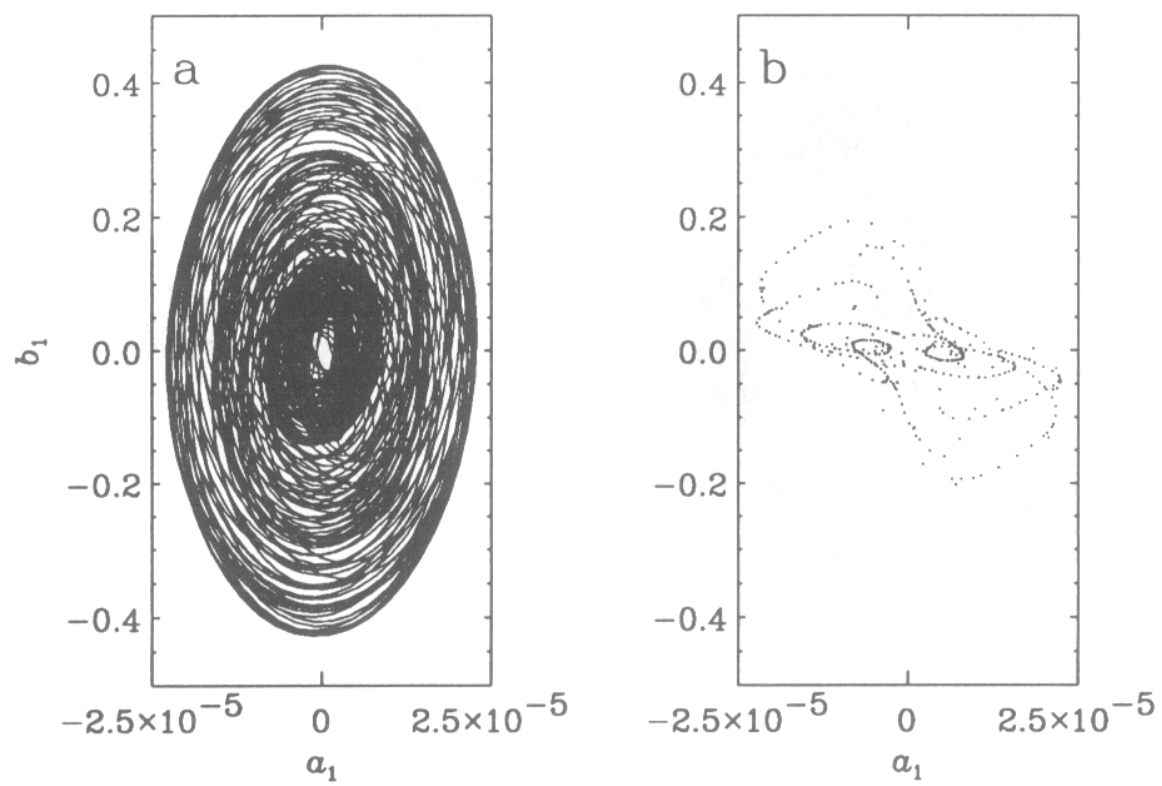

Fig. 6. (a) The phase portrait of the solution for $C_{\alpha}=-0.063$ projected onto the $A_{\phi}-B_{\phi}$-plane of a point at $(\varpi, z)=(0.52,-0.04)$ in the grid. (b) The Poincaré map of this solution. We plot the points where the $B_{\phi}$-field in $(0.52,0.04)$ goes through zero.
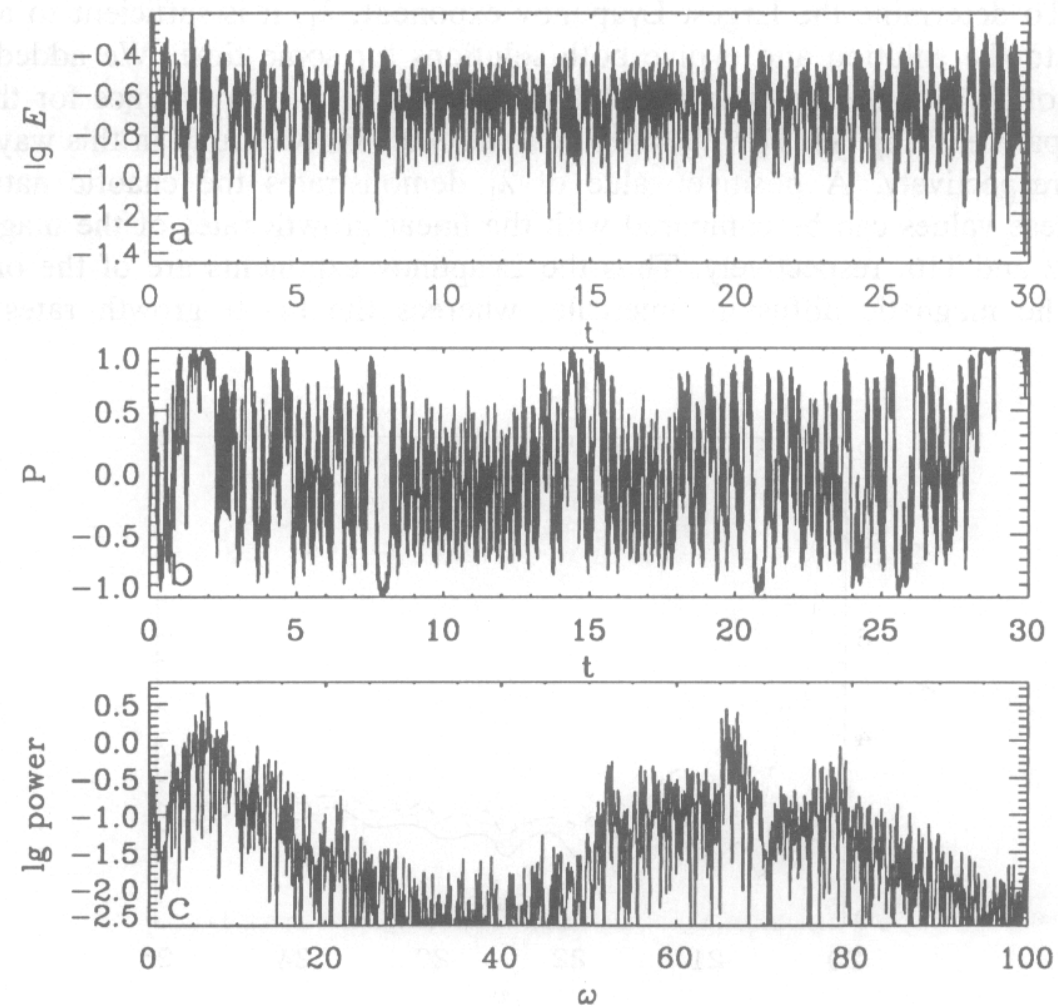

Fig. 7. The variations in $E$ (a) and $P$ (b) for an irregular dynamo solution with $\left|C_{\alpha}\right|=0.084$. The power spectrum of the oscillations in $E$ are displayed in (c). 

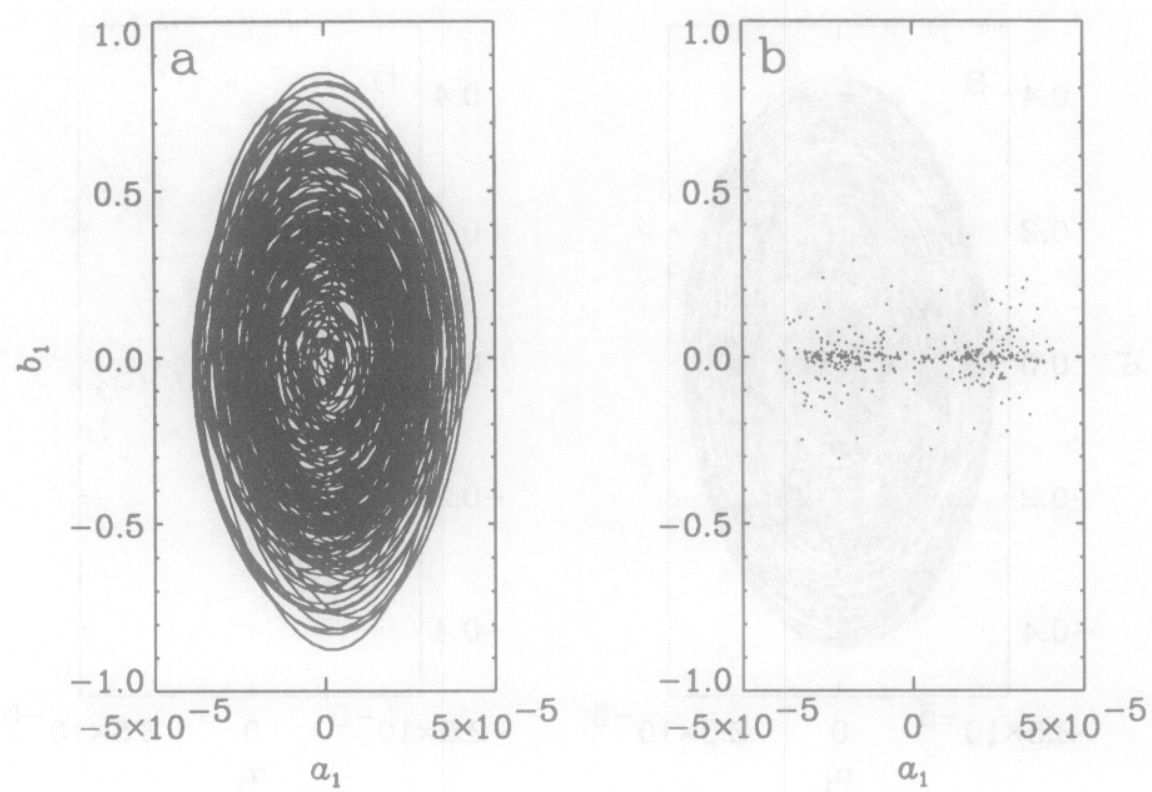

Fig. 8. (a) The phase portrait of the chaotic solution projected onto the $A_{\phi}-B_{\phi}$-plane of a point at $(\boldsymbol{w}, z)=(0.52,-0.04)$ in the grid. (b) The Poincare map of the same solution. We plot the points where the $B_{\phi}$-field in $(0.52,0.04)$ goes through zero.

To characterize the chaotic nature of a solution, it is of interest to calculate its Lyapunov exponents. To determine the largest Lyapunov exponent, $\lambda_{1}$, it is sufficient to add a small disturbance to the solution and evolve both solutions for some time. We added a relative disturbance of $10^{-6}$ to the $A_{\phi}$-field and determined the rate of divergence for the $B_{\phi^{-}}$and $A_{\phi}$-fields separately (Fig. 9). For $C_{\alpha}=-0.069$ and -0.084 , we found in this way $\lambda_{1} \approx+1.3$ and +2.5 , respectively. A positive value of $\lambda_{1}$ demonstrates the chaotic nature of the solution. These values can be compared with the linear growth rates of the magnetic field, which are 92 and 116, respectively. Thus the Lyapunov exponents are of the order of the inverse of the magnetic diffusion timescale, whereas the linear growth rates are much larger.

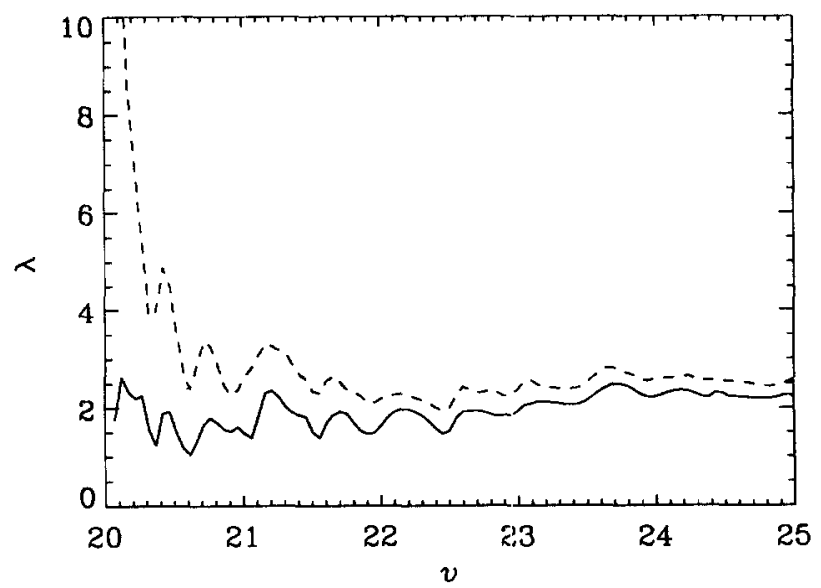

Fig. 9. The first cumulative Lyapunov exponent for $C_{\alpha}=-0.084$. The solid line is determined from the $A_{\phi}$-component, and the dashed line from the $B_{\phi}$-component. 
In order to estimate the dimension of the attractor, it would be of interest to determine the next largest Lyapunov exponents. We tried to estimate them both using a GramSchmidt orthonormalization of a bunch of nearby trajectories [7], and from a time series of the magnetic energy for a solution that was evolved for a long time [8]. So far, however, none of these techniques yielded reliable results in our case.

\section{DISCUSSION}

It is evident that results from linear calculations are misleading and insufficient for determining the magnetic field geometry in astrophysical bodies. For example, Moss and Tuominen [9] simulated nonlinear dynamo action in galactic disks and found the possibility of long-lived mixed-parity states. Complicated bifurcation diagrams including stable mixedparity solutions have been found in galactic disk-halo systems [10], and Brooke and Moss [11] studied the transition to chaos in a dynamo with torus geometry and Keplerian rotation.

There are similarities between the bifurcation sequence found in this paper and other nonlinear calculations. For a one-dimensional solar dynamo model, Jennings [12] found a steady $\mathrm{S} 0$-solution whose amplitude vanished for sufficiently large $C_{\alpha}$ while a new oscillatory A0-solution emerged. Similar behaviour was found by Brandenburg et al. [10] for a galactic dynamo. There is also a qualitative agreement in the form of the doubly-periodic oscillations of $P$ (Fig. 2 in [10]).

We should recall that an accretion disk has Keplerian rotation, leading to a stronger shear than in a galactic disk. Brooke and Moss [11] adopted such a Keplerian rotation law, but instead of a disk they employed a torus geometry. An interesting difference is that for So-solutions with positive $C_{\alpha}$, they only find steady solutions up to 30 times the critical value. This is a much larger interval than that in which we find a steady S0-solution. Nevertheless, they do find a gap with no dynamo excitation, but only in the linear theory. For negative $C_{\alpha}$ they find an oscillatory $\mathrm{A} 0$-solution, which later bifurcates to a doublyperiodic solution. This is in agreement with our result. When $\left|C_{\alpha}\right|$ increases further, the solution eventually begins to vary irregularly, however they do not find the second $T^{2}$ torus, as we do.

The route to chaos, $T^{2}\left(\rightarrow T^{3}\right) \rightarrow$ chaos $\rightarrow T^{2} \rightarrow$ chaos, suggested from our model, is similar to the bifurcation sequence found by Feudel et al. [13] for a rather different (solar dynamo) model. However, they do not find the intermediate chaotic state, which anyhow occurs only in a very narrow interval.

The disk model in our simulations is much thicker than real accretion disks, thus we cannot draw any quantitative conclusions regarding the behaviour of the magnetic fields of real accretion disks. However, it is worthwhile to draw a few qualitative conclusions. It is likely that $C_{\alpha}$ is positive in a real accretion disk, and it is therefore not clear whether our results of chaotically varying magnetic fields will occur in nature. On the other hand we find that for $C_{\alpha}>0$ the field is not always a steady quadrupole field as expected from linear theory. This is of great importance for theories of magnetohydrodynamic jet acceleration from accretion disks, because such mechanisms are usually more efficient for dipolar fields (e.g. [14]). An oscillatory magnetic field may also give rise to cyclic variations in an accretion disk, similar to the activity cycle of the Sun. Such variations may be observable through changes of the strength of emission lines (cf. [15]). Turning the whole argument around, we may gain a better understanding of the turbulent properties of accretion disks by observing magnetic fields in the disk. If no magnetic field exists, or if there is only a small-scale irregular magnetic field, we may obtain useful constraints on the turbulent properties of the disk. 
In conclusion, we have demonstrated that nonlinear effects may lead to the appearance and dominance of disk dynamo solutions other than the most easily excited ones. This is in particular the case for the steady S0-solution, which appears for the smallest dynamo number, but later it disappears again before any other mode has been excited. This gap of dynamo activity for certain dynamo numbers may be important for the properties of magnetic fields in accretion disks. It is also of interest for dynamo theory that we find both regularly oscillating and chaotically varying solutions of mixed parity, for certain parts of the parameter space.

Acknowledgements-The National Center for Atmospheric Research is sponsored by the National Science Foundation. The computations presented in this paper have been carried out mainly on the Cray X-MP 4/16 at the National Supercomputer Centre, Linköping, Sweden. We wish to thank the WE-Heraeus-Stiftung for financial support making the participation at this conference possible. We are also grateful to Ulrich Parlitz for determining Lyapunov exponents from a time series.

\section{REFERENCES}

1. U. Torkelsson and A. Brandenburg, Turbulent accretion disk dynamos, Astron. Astrophys. 283, 677 (1994).

2. N. I. Shakura and R. A. Sunyaev, Black holes in binary systems. Observational appearance, Astron. Astrophys. 24, 337 (1973).

3. G. Rüdiger, The alpha-effect in galaxies is highly anisotropic, Geophys. Astrophys. Fluid Dyn. 50, 53 (1990).

4. F. Krause and K.-H. Rädler, Mean-field Magnetohydrodynamics and Dynamo Theory. Pergamon, Oxford (1980).

5. F. Krause and R. Meinel, Stability of simple nonlinear $\alpha^{2}$-dynamos, Geophys. Astrophys. Fluid Dyn. 43, 95 (1988).

6. A. Brandenburg, F. Krause, R. Meinel, D. Moss and I. Tuominen, The stability of nonlinear dynamos and the limited role of kinematic growth rates, Astron. Astrophys. 213, 411 (1989).

7. A. Wolf, J. B. Swift, L. Swinney and J. A. Vastano, Determining Lyapunov exponents from a time series, Physica D, 16, 285 (1985).

8. U. Parlitz, Identification of true and spurious Lyapunov exponents from time series, J. Bifurcation Chaos 2, 155 (1992).

9. D. Moss and I. Tuominen, Nonlinear galactic dynamos, Geophys. Astrophys. Fluid Dyn. 50, 113 (1990).

10. A. Brandenburg, K. J. Donner, D. Moss, A. M. Shukurov, D. D. Sokoloff and I. Tuominen, Dynamos in discs and halos of galaxies, Astron. Astrophys. 259, 453 (1992).

11. J. M. Brooke and D. Moss, Nonlinear dynamos in torus geometry: Transition to chaos, Month. Not. Roy. Astron. Soc. 266, 733 (1994).

12. R. L. Jennings, Symmetry breaking in a nonlinear $\alpha \omega$-dynamo, Geophys. Astrophys. Fluid Dyn. 57, 147 (1991)

13. U. Feudel, W. Jansen and J. Kurths, Tori and chaos in a nonlinear dynamo model for solar activity, J. Bifurcation Chaos 3, 131 (1993).

14. J. C. L. Wang, M. E. Sulkanen and R. V. E. Lovelace, Self-collimated magnetic jets from magnetized accretion disks: The even symmetry case, Astrophys. J. 355, 38 (1990).

15. K. Horne and S. H. Saar, Rotation and emission lines in stars and accretion disks, Astrophys. J. 374, L55 (1991). 